\title{
SINDROME DE TAPIA BILATERAL APÓS CIRURGIA DE AVANÇO MAXILOMANDIBULAR
}

\section{RELATO DE CASO}

SILVA, Alexandre Augusto Ferreira da ${ }^{1}$, ROCHA, Roberto de Oliveira² ${ }^{2}$ MARIANO, Ronaldo Célio $^{3}$

SILVA, Alexandre Augusto Ferreira da. ROCHA, Roberto de Oliveira. MARIANO, Ronaldo Célio. Síndrome de Tapia bilateral após cirurgia de avanço maxilomandibular. Revista Científica Multidisciplinar Núcleo do Conhecimento. Ano. 06, Ed. 10, Vol. 08, pp. 49-59. Outubro 2021. ISSN: 2448-0959, Link de acesso: https://www.nucleodoconhecimento.com.br/saude/tapia-bilateral, DOI: 10.32749/nucleodoconhecimento.com.br/saude/tapia-bilateral

\section{RESUMO}

Lesões do nervo hipoglosso e laríngeo, recorrente em suas vias extracranianas unilaterais ou bilaterais, causam um déficit motor na língua com perda transitória, parcial ou total da fala, que pode estar associada à disfagia leve ou grave, caracterizando a síndrome de Tapia. Essa condição incomum costuma estar relacionada à manipulação das vias aéreas durante o ato anestésico-cirúrgico, sendo poucos os casos na literatura correlacionados com cirurgias maxilofaciais, o que explica, em parte, o desconhecimento dessa complicação por um número significativo de profissionais. Descrevemos um relato de caso de síndrome de Tapia bilateral estabelecida após procedimento para avanço maxilomandibular. Trata-se de um paciente do gênero masculino, 55 anos, com diagnóstico de retrognatismo e

\footnotetext{
${ }^{1}$ Mestre pelo Programa de Ciências Odontológicas da Universidade Federal de Alfenas (UNIFAL-MG). Residência em Cirurgia e Traumatologia Bucomaxilofacial pelo Hospital Central da Santa Casa de São Paulo-SP. Especialista e Membro Titular do Colégio Brasileiro de Cirurgia e Traumatologia Bucomaxilofacial.

${ }^{2}$ Doutor em medicina pela disciplina de Neurologia do Hospital das Clínicas da Faculdade de Medicina da Universidade de São Paulo.

${ }^{3}$ Orientador. Professor Titular da Faculdade de Odontologia da Universidade Federal de Alfenas (UNIFAL-MG). Doutor em Odontologia, Área de Cirurgia e Traumatologia Bucomaxilofacial pela Faculdade de Odontologia de Araçatuba, Unesp. Residência em Cirurgia e Traumatologia Bucomaxilofacial pela Faculdade de Odontologia de Ribeirão Preto, USP.
}

RC: 99979

Disponível em: https://www.nucleodoconhecimento.com.br/saude/tapia-bilateral 
retromaxilismo. O paciente foi submetido a um avanço maxilomandibular com intubação nasotraqueal. Evoluiu com síndrome de Tapia bilateral, recuperando-se dos sinais e sintomas desta complicação, sendo reoperado para corrigir resultados estéticos obtidos na primeira intervenção que estava abaixo do aceitável, não evoluindo após esta segunda cirurgia com a síndrome. Concluímos que medidas preventivas devem ser tomadas, como evitar movimentos bruscos na região da cabeça e hiperextensão prolongada do pescoço durante a cirurgia, minimizando o trauma da cânula traqueal sobre os tecidos que recobrem os nervos hipoglosso e laríngeo recorrente.

Palavras-chave: Síndrome, Nervo laríngeo recorrente, Nervo hipoglosso, Transtornos de deglutição, Disfonia.

\section{INTRODUÇÃO}

A Síndrome de Tapia é uma complicação extremamente rara relatada pela primeira vez em 1904 por Antonio Garcia Tapia, um médico otorrinolaringologista (VAREDI et al., 2003). Normalmente está associada à manipulação das vias aéreas para anestesia geral, caracterizada pelas lesões nos trajetos extracranianos do nervo hipoglosso (nervo motor da língua) e do nervo laríngeo recorrente (ramo do nervo vago que inerva a corda vocal), ocasionando neuropraxia destes nervos com maior frequência unilateral, porém, também podendo ser bilateral, comprometendo a movimentação da língua devido a lesão do nervo hipoglosso e dificuldade de fala pela lesão do nervo laríngeo recorrente (VAREDI et al., 2003; FARHAD; SAEID; HAMIDREZA, 2019). Cinar et al. (2005) acrescentam ainda aos sinais e sintomas, disfagia leve ou severa a depender da extensão da lesão dos nervos envolvidos e também com o fato do envolvimento ser uni ou bilateral, sendo que no envolvimento unilateral, dificuldade de fala associada a projeção da língua para fora da boca com desvio da sua ponta para o lado oposto a lesão são as características mais frequentes, enquanto que no comprometimento bilateral pode ocorrer completa ausência de protusão da língua e total incapacidade de deglutição em uma fase mais inicial da síndrome. 
O objetivo deste relato e descrever um caso que evoluiu com Síndrome de Tapia bilateral após cirurgia de avanço maxilomandibular. Depois do paciente recuperar-se dos sinais e sintomas da síndrome, o mesmo precisou ser reoperado, não evoluindo após a segunda intervenção com a Síndrome de Tapia.

\section{RELATO DO CASO}

Paciente de 55 anos de idade, gênero masculino, leucoderma, com diagnóstico de retrognatismo com assimetria mandibular e retromaxilismo com deficiência vertical de maxila, associados com quadro clínico de apneia obstrutiva do sono em grau moderado. Os motivos que levaram o paciente a procurar tratamento eram roncos, sonolência diurna e estética facial comprometida. O paciente foi submetido a uma anestesia geral com intubação nasotraqueal para a realização de uma cirurgia esquelética de avanço de $12 \mathrm{~mm}$ de maxila e mandíbula.

O paciente, apesar da sua deformidade esquelética maxilofacial, foi considerado de provável intubação fácil, porém, somente durante a segunda tentativa de laringoscopia procedeu-se a intubação. O cuff do tubo nasotraqueal foi inflado com pressão inferior a $20 \mathrm{~cm} \mathrm{H}_{2} \mathrm{O}$. Foi utilizado um tampão orofaríngeo correspondente ao volume de três gazes de $7,5 \mathrm{~cm} \times 7,5 \mathrm{~cm}$ de tamanho, para minimizar a passagem de fluidos para o estomago. Durante o ato operatório que durou 180 minutos houve a necessidade de insuflar novamente o cuff que havia perdido pressão, tomando o cuidado de não exceder os $20 \mathrm{~cm}$ de $\mathrm{H}_{2} \mathrm{O}$. Uma hipotensão arterial sistólica foi estabelecida em aproximadamente 90 a $100 \mathrm{mmHg}$ durante toda a cirurgia. $\mathrm{O}$ paciente permaneceu em posição semi-supina com a cabeça levemente mais elevada que o tronco.

Após o término da cirurgia o paciente foi extubado sem nenhum tipo de intercorrência. Quarenta e oito horas após a cirurgia o paciente ainda não conseguia falar e diagnosticamos que a língua quase não apresentava movimentação no sentido de protruir para fora da boca, lateralizar-se ou elevar-se até o contato com o palato. Nesta ocasião foi verificado também que a deglutição estava muito comprometida e o paciente não conseguia engolir sua própria saliva. Uma 
nasofibroscopia foi realizada onde pode se constatar paralisia das cordas vocais bilaterais. Devido ao quadro de dificuldade de deglutição, a dieta via oral foi suspensa e uma sonda nasogastrica foi colocada para nutrição do paciente. Quatro dias após a cirurgia o quadro não apresentava nenhuma alteração. Exame de tomografia computadorizada de crânio não identificou alterações significativas e ressonância magnética de crânio e pescoço verificou que o trajeto intracraniano do nervo hipoglosso, bem como seu canal e o forame jugular estavam preservados. Não foi identificado também alterações da topografia do nervo hipoglosso no seu trajeto extracraniano. Após estar descartado qualquer tipo de envolvimento do sistema nervoso central foi estabelecido o diagnóstico de Síndrome de Tapia associada a um quadro de disfagia e disfonia severas. Iniciou-se a administração de corticoides (dexametasona - 4mg endovenoso de 8 em 8 horas durante 2 semanas) e de vitaminas do complexo B durante dois meses com o objetivo de acelerar a reparação dos nervos lesados. Tão logo o edema pós-operatório começou a regredir iniciou-se terapia fonoaudiológica para fala e deglutição. O quadro clínico do paciente permaneceu inalterado em relação à Síndrome de Tapia durante a primeira semana de pós-operatório e, ao final desta, foi diagnosticada uma pneumonia causada por aspiração de alimentos líquidos durante as primeiras 48 horas do pósoperatório, quando a dieta liquida via oral ainda estava mantida. Ao longo das semanas subsequentes o paciente se recuperou totalmente da pneumonia, sendo que somente com três semanas de pós-operatório era possível observar que o lado direito da língua começava a apresentar um pequeno movimento de protusão, com desvio da ponta da língua para a esquerda, fato este que até então não se observava, mas sem capacidade alguma de elevar a ponta da língua de encontro ao palato (Figura 1). Nesta ocasião também o paciente começou a falar com muita rouquidão e extrema dificuldade. A cada semana o paciente melhorava um pouco a movimentação da língua e a qualidade da voz, mesmo tendo que alimentar-se por sonda nasogastrica e, somente com dois meses de pós-operatório, após vários testes realizados pela fonoaudióloga para verificar se o paciente estava conseguindo deglutir e não aspirando alimentos pastosos, a sonda nasogastrica foi removida e a nutrição oral foi restabelecida, sem riscos de uma nova aspiração com desenvolvimento de uma nova pneumonia aspirativa. O paciente seguiu com terapia 
fonoaudiológica de motricidade oral e para melhora da fala e da deglutição, sendo que o progresso foi lento, mas constante, até que com quatro meses de pósoperatório o paciente já conseguia fazer todos os movimentos da língua (Figura 2), tais como: protruir para fora da boca, fazer lateralidade e elevar a ponta da língua de encontro ao palato. A voz apresentava melhora significativa da rouquidão, mas ainda assim estava muito diferente em relação ao pré-operatório. Nova nasofibroscopia evidenciou agora somente paralisia da corda vocal esquerda.

O paciente continuou apresentando melhora significativa dos sinais e sintomas da Síndrome de Tapia a cada mês que passava, porém, o resultado estético esperado da cirurgia de avanço esquelético maxilomandibular ficou muito aquém do que havia sido planejado. Então com dezoito meses de pós-operatório, com todos os movimentos da língua recuperados e ainda com uma pequena rouquidão na voz, o paciente foi reoperado.

Estabelecemos cuidados para diminuir as chances de uma recidiva dos sinais e sintomas desta síndrome, como: realizar a intubação nasotraqueal com o mínimo trauma possível, controlar a pressão no cuff da sonda, não usar tampão orofaríngeo, diminuir o máximo possível o tempo cirúrgico e evitar manipulação excessiva ou hiperextensão do pescoço durante a cirurgia. Desta vez realizamos uma cirurgia somente na maxila associada a uma mentoplastia, cujo objetivo foi diminuir a extensão e a duração da cirurgia. O paciente foi então novamente submetido, após indução venosa e curarização profunda, a uma intubação nasotraqueal com mínima extensão cervical e mínima manipulação da glote, obtendo-se êxito na primeira tentativa. Como na primeira cirurgia, o paciente foi mantido em posição semi-supina com a cabeça levemente mais elevada que o tronco e o tampão orofaríngeo desta vez não foram utilizados com o objetivo de prevenir compressão sobre os nervos hipoglosso e laríngeo recorrente. A cirurgia teve uma duração de 120 minutos e ao término da cirurgia o paciente foi extubado com mínima manipulação da via aérea sem uso de cânula orofaríngea. Imediatamente após a retirada do tubo nasotraqueal o paciente já era capaz de pronunciar várias palavras e, quando solicitado para movimentar a língua em todas as direções, assim o fez. O jejum absoluto foi mantido 
nas primeiras doze horas de pós-operatório. No pós-operatório de vinte e um meses da primeira cirurgia e de três meses da segunda cirurgia, novo exame de nasofibroscopia evidenciou ainda persistência da paralisia da corda vocal esquerda, sendo que o paciente ainda apresenta uma leve rouquidão na voz, que não foi agravada pela segunda intervenção e que não atrapalha seu convívio social, permanecendo ainda em terapia fonoaudiológica para melhora da fala. Os resultados estéticos que motivaram a indicação da segunda cirurgia foram considerados adequados pela equipe e pelo paciente.

\section{DISCUSSÃO}

A Síndrome de Tapia, embora extremamente rara, tem como causa mais provável, a manipulação das vias aéreas em pacientes submetidos à anestesia geral, seja por intubação naso ou orotraqueal (CINAR et al.,2005; NALLADARU; WESSELS; DUPREEZ, 2012; CARIATI et al., 2016; MUMTAZ; HENRY; SINGH, 2018; FARHAD; SAEID; HAMIDREZA, 2019). Ota et al. (2013) descreveram a etiologia da Síndrome de Tapia como sendo um trauma mecânico, produzindo um dano sobre o nervo laríngeo recorrente, ramo do nervo vago, e sobre o nervo hipoglosso, que se encontram próximo da base da língua, sendo que esse local onde os nervos vago e hipoglosso atravessam pode ser comprimido pelo tubo endotraqueal.

Há relatos de Síndrome de Tapia após cirurgias em outras localizações fora da cabeça e pescoço, como por exemplo, após cirurgia cardíaca (NALLADARU; WESSELS; DUPREEZ, 2012). Há casos relatados cuja proximidade do tubo traqueal com o sítio da cirurgia é muito grande, como por exemplo, após reparação de fratura de mandíbula, rinoplastia, cirurgia para fixação de fratura de zigoma, cirurgia ortognática e remoção de terceiro molar superior retido sob anestesia geral (KASHYAP et al., 2010; LYKOUDIS; SERETIS, 2012; VAREDI et al., 2013; OTA et al., 2013; MUMTAZ; HENRY; SINGH, 2018). Em cirurgias realizadas na face é comum durante o transoperatório o cirurgião corrigir, uma ou mais vezes, o posicionamento da cabeça do paciente, ou mesmo virá-la mais para um lado ou para o outro a depender da região que ele irá atuar, sendo que isto pode, em algumas 
circunstâncias, promover o deslocamento do tubo traqueal e este vir a traumatizar a região superficialmente aos nervos envolvidos nesta síndrome (OTA et al., 2013). Portanto, medidas preventivas como uma intubação com menos trauma possível,

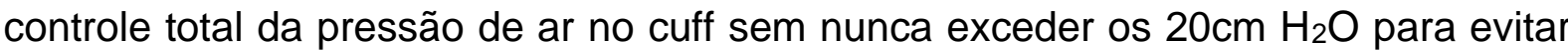
compressão excessiva dos tecidos superficialmente aos nervos, posicionamento adequado da cabeça do paciente sem hiperextensão do pescoço por longo tempo, evitando movimentos bruscos da cabeça durante a cirurgia, podem contribuir para a prevenção desta síndrome.

A incidência bilateral da Síndrome de Tapia é excepcionalmente rara, sendo que encontramos na literatura o relato de caso de Cinar et al. (2005) que descreveram uma ocorrência bilateral desta síndrome após uma rinoplastia, com resolução dos sintomas completamente em quatro semanas. No presente relato, também com envolvimento bilateral, o paciente somente começou a movimentar totalmente a língua e teve a sonda nasogastrica removida com dois meses de pós-operatório da primeira cirurgia, e com 18 meses de pós-operatório o paciente ainda apresentava paralisia da corda vocal esquerda e rouquidão leve na voz que não o incomodava, sendo então reoperado, não apresentando neste segundo pós-operatório, os sinais e sintomas da Síndrome de Tapia, demostrando que os cuidados de prevenção são estratégias fundamentais para evitar esta condição.

A paralisia dos nervos hipoglosso e laríngeo recorrente de forma unilateral acarretam os sinais clássicos da Síndrome de Tapia que são: déficit motor na língua com desvio da ponta para o lado oposto ao nervo hipoglosso lesado durante o movimento de protusão da mesma e disfonia devido à paralisia da corda vocal ipsilateral (KASHYAP et al., 2010; LYKOUDIS; SERETIS, 2012). No entanto, vários autores acrescentam que a disfagia pode estar presente em casos em que o trauma nos nervos envolvidos tenha sido maior, podendo acontecer em situações unilaterais e bilaterais (CINAR et al.,2005; VAREDI et al., 2013; CARIATI et al., 2016). Nos casos bilaterais há quase completa ausência de movimentação da língua em todas as direções, como no caso aqui relatado. Ibrahim e Sarper (2010) classificou a Síndrome de Tapia em suave, moderada ou severa, entretanto, consideraram 
apenas o envolvimento unilateral da síndrome, sendo que somente no tipo severo o paciente apresentava dificuldades na deglutição. No presente caso, até por ser bilateral, o paciente teve total incapacidade de engolir sua própria saliva durante 0 primeiro mês de pós-operatório da primeira cirurgia.

Para o tratamento desta complicação indesejável é sugerido realizar a terapia com corticoide tão logo o diagnóstico seja feito por um período que pode variar em torno de duas semanas, bem como a administração de vitaminas do complexo B, objetivando uma reparação mais precoce da função dos nervos envolvidos, até que ocorra melhora importante dos sintomas (CINAR et al.,2005; LYKOUDIS; SERETIS, 2012; CARIATI et al., 2016; MUMTAZ; HENRY; SINGH, 2018). Há um consenso que a terapia fonoaudiológica para estimular a melhora da dicção, da movimentação da língua e da disfagia, quando presente, seja fundamental para auxiliar no reestabelecimento do paciente (VAREDI et al., 2013; MUMTAZ; HENRY; SINGH, 2018; FARHAD; SAEID; HAMIDREZA, 2019).

Torna-se importante enfatizar a necessidade de um diagnóstico precoce da Síndrome de Tapia, ainda nas primeiras horas do pós-operatório em que o paciente tenha sido submetido à anestesia geral, uma vez que em casos onde a disfagia possa estar presente, a liberação da dieta pode levar a outras complicações, como ocorreu no presente caso, onde o paciente permaneceu com dieta liquida nas primeiras 48 horas, aspirando conteúdo para os pulmões com consequente desenvolvimento de uma pneumonia, que complicou consideravelmente a sua recuperação. Tão logo o paciente recupere sua consciência após a anestesia geral e antes de introduzir a dieta é fundamental checar a movimentação da língua em todas as direções, bem como se o paciente consegue pronunciar algumas palavras e deglutir sua própria saliva. Pela sua casuística extremamente incomum a Síndrome de Tapia é desconhecida da grande maioria dos profissionais, fazendo com que o seu diagnóstico seja retardado até que toda equipe que acompanha o paciente consiga compreender melhor os sinais e sintomas apresentados pelo paciente, de tal forma que o diagnóstico possa ser elaborado e consequentemente $o$ 
tratamento introduzido. No presente caso o diagnóstico foi tardio e dificultado por tentar encontrar relação dos sinais e sintomas com a cirurgia realizada.

\section{CONSIDERAÇÕES FINAIS}

As cirurgias de face têm uma relação extremamente baixa com o desenvolvimento da Síndrome de Tapia, desde que medidas preventivas sejam tomadas, como evitar movimentos bruscos na região da cabeça e a hiperextensão do pescoço por longos períodos durante a cirurgia, minimizando o trauma do tubo traqueal sobre os tecidos que recobrem os nervos hipoglosso e laríngeo recorrente.

\section{REFERÊNCIAS}

CARIATI, Paolo; CABELLO Almudena; GALVEZ, Pablo P; LOPEZ, Dario Sanchez; MEDINA, Blas Garcia. Tapia's syndrome: pathogenetic mechanisms, diagnostic management, and proper treatment: a case series. J Med Case Rep. v. 10, n. 23, jan. 2016. https://doi.org/10.1186/s13256-016-0802-1

CINAR SO, SEVEN H, CINAR U, TURGUT S. Isolated bilateral paralysis of the hypoglossal and recurrent laryngeal nerves (Bilateral Tapia's syndrome) after transoral intubation for general anesthesia. Acta Anaesthesiol Scand. v. 49, n. 1, p. 98-99, jan. 2005. https://doi.org/10.1111/j.1399-6576.2004.00553.x

FARHAD, Ghorbani; SAEID Tavanafar; HAMIDREZA Eftekharian. Tapia's syndrome after cosmetic malar augmentation: a case report. J Dent Shiraz Univ Med Sci. v. 20, n.1, p. 66-70, mar. 2019. doi: https://doi.org/10.30476/DENTJODS.2019.44566

IBRAHIM, Boga; SARPER Aktas. Treatment, classification, and review of Tapia syndrome. J Craniofac Surg. v. 21, n.1, p. 278-280, jan. 2010. https://doi.org/10.1097/SCS.0b013e3181c678f0

KASHYAP, Soumya A; PATTERSON, Alan R; LOUKOTA, Richard A; KELLY, Gerard. Tapia's syndrome after a repair of a fractured mandible. $\mathbf{B r} \mathbf{J}$ Oral 
Maxillofac Surg. v. 48, n. 1, p. 53-54, jan. 2010. https://doi.org/10.1016/j.bjoms.2009.01.021

LYKOUDIS, Efstathios G; SERETIS, Konstantinos. Tapia's syndrome: An unexpected but real complication of rhinoplasty: case report and literature review. Aesthetic Plastic Surg. v. 36, n. 3, p. 557-559, jun. 2012. https://doi.org/10.1007/s00266-011-9849-y

MUMTAZ, Shadaab; HENRY, Alastair; SINGH, Mark. Tapia's syndrome. Anesth Prog. v. 65, n. 2, p. 129-130, jun. 2018. https://doi.org/10.2344/anpr-65-04-06

NALLADARU, Zubin; WESSELS, Andre; DUPREEZ, Leon. Tapia's syndrome - a rare complication following cardiac surgery. Interact Cardiovasc Thorac Surg. v. 14, n. 1, p. 131-132, jan. 2012. https://doi.org/10.1093/icvts/ivr056

OTA, Nobutaka; IZUMI, Kiwako; OKAMOTO, Yoshihiko; TOSHITANI, Koji; NAKAYAMA, Keisuke; FUKUZAWA, Hideaki; OZEKI, Satoru; IKEBE, Tetsuro. Tapia's syndrome following the orthognatic surgery under general anaesthesia. $\mathbf{J}$ Oral Maxillofac Surg Med Pathol. v. 25, n. 1, p. 52-54, jan. 2013. https://doi.org/10.1016/j.ajoms.2012.02.001

VAREDI, Payam; SHIRANI, Gholamreza; KARIMi, Abbas; VAREDI, Peyman; KHIABANI, Kazem; BOHLULI, Behnam. Tapia syndrome after repairing a fractured zygomatic complex: a case report and review of the literature. J Oral Maxillofac $\begin{array}{lllllll}\text { Surg. } & \text { v. } 71, \quad \text { n. } 10, & \text { p.1665-1669, }\end{array}$ https://doi.org/10.1016/j.joms.2013.05.019 


\section{FIGURAS E LEGENDAS}

Figura 1: Pós-operatório de três semanas da primeira cirurgia onde começamos a observar uma pequena projeção da língua para fora da boca com desvio da ponta para a esquerda.

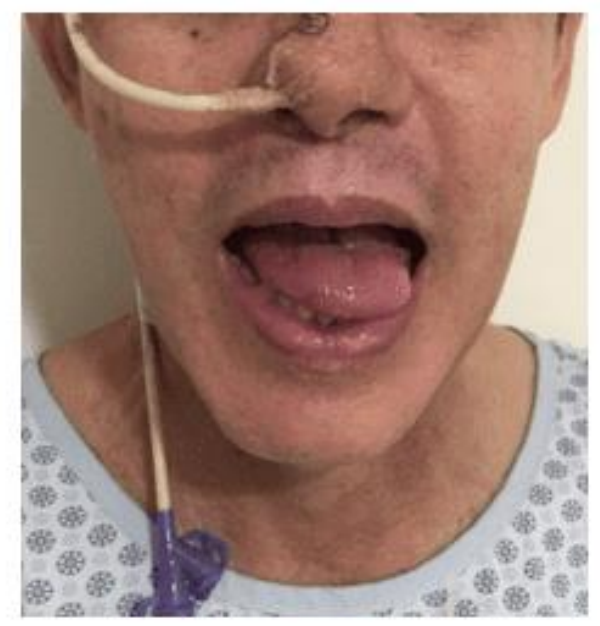

Fonte: autor.

Figura 2: Pós-operatório de 4 meses da primeira cirurgia quando todos os movimentos da língua foram recuperados.

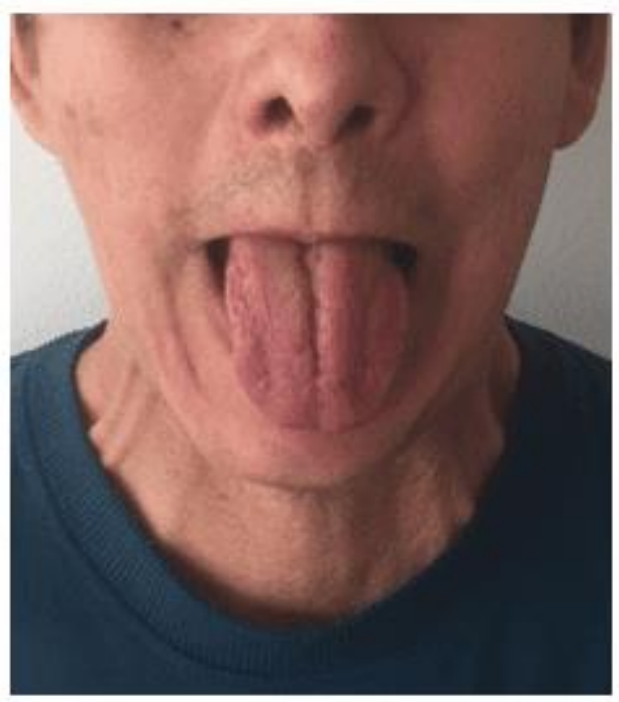

Fonte: autor.

RC: 99979

Disponível em: https://www.nucleodoconhecimento.com.br/saude/tapia-bilateral 
Enviado: Agosto, 2021.

Aprovado: Outubro, 2021.

RC: 99979

Disponível em: https://www.nucleodoconhecimento.com.br/saude/tapia-bilateral 